\title{
An integral characterization of Hajłasz-Sobolev space
}

\section{Marc TROYANOV ${ }^{\text {a }}$, Vladimir GOL'DSHTEIN ${ }^{\text {b }}$}

a Départment de mathématiques, EPFL, CH-1015 Lausanne, Suisse

${ }^{b}$ Departement of Mathematics, Ben Gurion University of The Negev, P.O. Box 653, 84105 Beer Sheva, Israel

E-mail: marc.troyanov@epfl.ch; vladimir@bgumail.bgu.ac.il

(Reçu et accepté le 10 juillet 2001)

\begin{abstract}
We prove that the pointwise inequality used by P. Hajłasz in his definition of Sobolev spaces on metric spaces is equivalent to an integral (Poincaré-type) inequality. ( 2001 Académie des sciences/Éditions scientifiques et médicales Elsevier SAS
\end{abstract}

\section{Une caractérisation intégrale de l'espace de Hajłasz-Sobolev}

Résumé. $\quad$ On démontre dans cette Note que l'inégalité ponctuelle utilisée par Piotr Hajtasz dans sa définition des espaces de Sobolev sur les espaces métriques mesurés est équivalente à une inégalité intégrale du type de l'inégalité de Poincaré. () 2001 Académie des sciences/Éditions scientifiques et médicales Elsevier SAS

\section{Version française abrégée}

Dans l'article [4], P. Hajłasz associe à tout espace métrique mesuré $(X, d, \mu)$ l' «espace de Sobolev» $M^{1, p}(X)$ constitué par l'ensemble des fonctions $u \in \mathrm{L}^{p}(X, \mu)$ pour lesquelles il existe une fonction $g \in \mathrm{L}^{p}(X, \mu)$ telle que

$$
|u(x)-u(y)| \leqslant(g(x)+g(y)) d(x, y)
$$

presque partout.

Nous démontrons dans cette Note que si la mesure $\mu$ possède la propriété locale de dédoublement (i.e. il existe $\alpha<\infty$ tel que, pour tout $x \in X$ et tout $r<1$, on a $\mu\left(\mathrm{B}(x, 2 r) \leqslant 2^{\alpha} \mu(\mathrm{B}(x, r))\right.$, alors l'espace de Sobolev $M^{1, p}(X)$ peut aussi être défini en remplaçant l'inégalité ci-dessus par une inégalité de type Poincaré :

THÉORÈme. - Supposons que $\mu$ possède la propriété locale de dédoublement et que $\mu(\{x\})=0$ pour tout $x \in X$, alors pour toute paire de fonctions $(u, g)$ telle que $u \in \mathrm{L}_{\mathrm{loc}}^{1}(X)$ et $g \in \mathrm{L}^{p}(X)$, les conditions suivantes sont équivalentes:

(a) il existe une constante $C$ telle que l'inégalité

$$
|u(x)-u(y)| \leqslant C(g(x)+g(y)) d(x, y)
$$

est vérifiée pour tous $x$, y appartenant au complémentaire d'un ensemble de mesure nulle;

\section{Note présentée par Haïm BREZIS.}




\section{Troyanov, V. Gol'dshtein}

(b) il existe une constante $C^{\prime}$ telle que, pour toute partie mesurable bornée $A \subset X$, on a

$$
f_{A}\left|u-u_{A}\right| \mathrm{d} \mu \leqslant C^{\prime} \operatorname{diam}(A)\left(f_{A} g^{p} \mathrm{~d} \mu\right)^{1 / p} .
$$

Ce résultat est encore vrai avec des hypothèses un peu plus générales; il suffit en fait que la mesure soit quasi homogène et qu'elle vérifie la propriété de Lebesgue (voir ci-dessous).

\section{Introduction}

Let $(X, d)$ be a metric space equipped with a Borel measure $\mu$ such that $0<\mu(B)<\infty$ for any metric ball $B \subset X$ of positive radius. The associated Hajłasz-Sobolev space $M^{1, p}(X)$ is defined to be the set of functions $u \in \mathrm{L}^{p}(X)$ for which there exists $g \in \mathrm{L}^{p}(X)$ such that

$$
|u(x)-u(y)| \leqslant(g(x)+g(y)) d(x, y)
$$

almost everywhere.

For any $1 \leqslant p<\infty$, the space $M^{1, p}(X)$ is a Banach space for the norm

$$
\|u\|_{M^{1, p}(X)}:=\|u\|_{\mathrm{L}^{p}(X)}+\inf _{g}\|g\|_{\mathrm{L}^{p}(X)},
$$

where the infimum is being taken over all functions $g$ satisfying (1). It has been proved by P. Hajłasz in [4] that, if $\Omega \subset \mathbb{R}^{n}$ is a domain with regular boundary and $p>1$, then the Hajłasz-Sobolev space coincides with the classic Sobolev space, i.e., $M^{1, p}(\Omega)=\mathrm{W}^{1, p}(\Omega)$.

This result suggests that a pair of function $(u, g)$ satisfying (1) is a reasonable generalization for the notion of a pair $(u,|\nabla u|)$ in the context of measure metric spaces; ${ }^{1}$ however, there are other useful generalizations of this notion (see, e.g., [3]). In particular, one says that a pair $(u, g)$ satisfies a $(1, p)$ Poincaré inequality if there exists two constants $\sigma \geqslant 1$ and $C>0$ such that

$$
f_{\mathrm{B}(x, r)}\left|u-u_{B}\right| \mathrm{d} \mu \leqslant C r\left(f_{\mathrm{B}(x, \sigma r)} g^{p} \mathrm{~d} \mu\right)^{1 / p}
$$

for any ball $\mathrm{B}(x, r) \subset X$ (we use the classical notation $u_{A}$ for the average of a function $u$ on a set $A$ :

$$
\left.u_{A}=f_{A} u \mathrm{~d} \mu=\frac{1}{\mu(A)} \int_{A} u \mathrm{~d} \mu\right) .
$$

Recent work has shown that a large part of analysis on metric spaces can be developed from the Poincaré inequality only (assuming some condition on the measure $\mu$ ) (see in particular [1,5] and references therein).

It is not difficult to show that any pair of functions $(u, g)$ satisfying (1) also satisfies a $(1, p)$-Poincaré inequality, it follows in particular from the theorem below. Our goal in this note is to prove a result in the converse direction. We need first a few measure theoretic definitions.

\section{Quasi-homogeneous measures}

DEFINITION 1. - The measure $\mu$ has locally the doubling property if there exist two constants $\alpha, \eta>0$ such that, for almost all $x \in X$, we have

$$
\mu(\mathrm{B}(x, 2 r)) \leqslant 2^{\alpha} \mu(\mathrm{B}(x, r))
$$

for all $r<\eta$.

Graphs, Riemannian manifolds with bounded geometry and Carnot groups are simple examples of locally doubling measure metric spaces. 
DEFINITION 2. - The measure $\mu$ satisfies the Lebesgue property if, for any $f \in \mathrm{L}_{\text {loc }}^{1}(X, \mu)$, the condition

$$
f(x)=\lim _{r \rightarrow 0} f_{\mathrm{B}(x, r)} f \mathrm{~d} \mu
$$

holds almost everywhere. Those points where this conditions holds are called the Lebesgue points of $f$.

It is known that every locally doubling measure satisfies the Lebesgue property (see, e.g., [5]).

Definition 3. - The measure $\mu$ on the metric space $(X, d)$ is quasi-homogeneous if there exists $K>0$ and $X_{0} \subset X$ such that $\mu\left(X \backslash X_{0}\right)=0$ and for any $x, y \in X_{0}$ we can find two sequences of positive numbers $\left\{s_{k}\right\}$ and $\left\{t_{k}\right\}$, such that $s_{k} \rightarrow 0, t_{k} \rightarrow 0$ and

$$
K^{-1} \leqslant \frac{\mu\left(\mathrm{B}\left(x, t_{k}\right)\right)}{\mu\left(\mathrm{B}\left(y, s_{k}\right)\right)} \leqslant K .
$$

An example of quasi-homogeneous measure is given by a measure on a discrete metric space $X$ such that, for any point $x \in X$, we have $0<C_{1} \leqslant \mu(\{x\}) \leqslant C_{2}<\infty$.

Other examples are provided by the following:

Lemma. - Suppose that the measure $\mu$ is atomfree and that, for a.e. $x \in X$, there exists a decreasing sequence $\left\{r_{m}(x)\right\}$ such that $\lim _{m \rightarrow \infty} r_{m}=0$ and

$$
\mu\left(\mathrm{B}\left(x, r_{m}(x)\right)\right) \leqslant C \mu\left(\mathrm{B}\left(x, r_{m+1}(x)\right)\right)
$$

for all $m \geqslant 1$ where the constant $C \geqslant 1$ is independent of $x$. Then $\mu$ is quasi-homogeneous.

It follows easily from this lemma that any atomfree measure satisfying one of the following conditions:

a) the function $r \rightarrow \mu(\mathrm{B}(x, r))$ is continuous near $r=0$ for a.e. $x \in X$, or

b) the measure $\mu$ is locally doubling,

is quasi-homogeneous.

Recall that a measure $\mu$ is atomfree if $\mu(\{x\})=0$ for all $x \in X$; equivalently, $\lim _{r \rightarrow 0} \mu(\mathrm{B}(x, r))=0$ for all $x$.

Proof. - Let us fix $x, y \in X$ and $k \geqslant 1$. Set $s_{k}:=\min \left\{r_{k}(x), r_{k}(y), 1 / k\right\}$ and assume that $\mu\left(\mathrm{B}\left(x, s_{k}\right)\right) \geqslant$ $\mu\left(\mathrm{B}\left(y, s_{k}\right)\right)$. Because $\lim _{t \rightarrow 0} \mu(\mathrm{B}(x, t))=0$, we can find an integer $m \geqslant 1$ such that

$$
\mu\left(\mathrm{B}\left(x, r_{m}(x)\right)\right) \geqslant \mu\left(\mathrm{B}\left(y, s_{k}\right)\right)>\mu\left(\mathrm{B}\left(x, r_{m+1}(x)\right)\right) .
$$

By hypothesis we have

$$
\mu\left(\mathrm{B}\left(x, r_{m+1}(x)\right)\right) \geqslant \frac{1}{C} \mu\left(\mathrm{B}\left(x, r_{m}(x)\right)\right),
$$

hence if we set $t_{k}:=r_{m}$ we have

$$
\frac{1}{C} \leqslant \frac{\mu\left(\mathrm{B}\left(y, s_{k}\right)\right)}{\mu\left(\mathrm{B}\left(x, t_{k}\right)\right)} \leqslant 1 .
$$

In case $\mu\left(\mathrm{B}\left(x, s_{k}\right)\right) \leqslant \mu\left(\mathrm{B}\left(y, s_{k}\right)\right)$, we obtain

$$
1 \leqslant \frac{\mu\left(\mathrm{B}\left(y, s_{k}\right)\right)}{\mu\left(\mathrm{B}\left(x, t_{k}\right)\right)} \leqslant C ;
$$

this concludes the proof.

An example of measure which is not quasi-homogeneous is given by a measure containing atoms (i.e., points $x$ such that $\mu(\{x\})>0)$ together with an atomfree part of positive measure. 


\section{Troyanov, V. Gol'dshtein}

\section{The main result}

THEOREM 4. - If $\mu$ is quasi-homogeneous and satisfies the Lebesgue property, then for any locally integrable function $u$ on $X$ and any $g \in \mathrm{L}^{p}(X)(1 \leqslant p<\infty)$ the following conditions are equivalent:

(a) there exists a constant $C$ such that the inequality

$$
|u(x)-u(y)| \leqslant C(g(x)+g(y)) d(x, y)
$$

holds for any $x, y$ in the complement of a subset of measure zero;

(b) there exists a constant $C^{\prime}$ such that for any bounded measurable subset $A \subset X$ we have

$$
f_{A}\left|u-u_{A}\right| \mathrm{d} \mu \leqslant C^{\prime} \operatorname{diam}(A)\left(f_{A} g^{p} \mathrm{~d} \mu\right)^{1 / p} .
$$

Proof. - (a) $\Rightarrow$ (b) follows from a double integration of the inequality (3) (see [4] or [6]).

To prove (b) $\Rightarrow($ a), we consider a pair $(u, g)$ satisfying the inequality (4) for any bounded measurable subset $A \subset X$.

Let $X_{0}$ be the set given in Definition 3, and observe that we may furthermore assume without lost of generality that any point in $X_{0}$ is a Lebesgue point for both functions $u$ and $g^{p}$.

Choose two distinct points $x, y \in X_{0}$ and let us set $B_{k}:=\mathrm{B}\left(x, t_{k}\right)$ and $B_{k}^{\prime}:=\mathrm{B}\left(y, s_{k}\right)$ where $t_{k}, s_{k}$ are as in Definition 3.

Since $\mu\left(B_{k}\right) / \mu\left(B_{k}^{\prime}\right)$ is a bounded sequence, we may assume (passing to a subsequence if necessary) that the limit

$$
\ell:=\lim _{k \rightarrow \infty} \frac{\mu\left(B_{k}\right)}{\mu\left(B_{k}^{\prime}\right)}
$$

exists. Observe then that $K^{-1} \leqslant \ell \leqslant K$ where $K$ is the constant in Definition 3.

Set $A_{k}:=B_{k} \cup B_{k}^{\prime}$; if $k>2 / d(x, y)$, then $B_{k} \cap B_{k}^{\prime}=\varnothing$ and $A_{k}$ is thus the disjoint union of $B_{k}$ and $B_{k}^{\prime}$. It follows that

$$
u_{A_{k}}=\frac{1}{\mu\left(A_{k}\right)} \int_{A_{k}} u \mathrm{~d} \mu=\frac{\mu\left(B_{k}\right)}{\mu\left(A_{k}\right)}\left(\frac{1}{\mu\left(B_{k}\right)} \int_{B_{k}} u \mathrm{~d} \mu\right)+\frac{\mu\left(B_{k}^{\prime}\right)}{\mu\left(A_{k}\right)}\left(\frac{1}{\mu\left(B_{k}^{\prime}\right)} \int_{B_{k}^{\prime}} u \mathrm{~d} \mu\right) .
$$

Let us set $\lambda=\frac{\ell}{1+\ell}$, then we have

$$
\lim _{k \rightarrow \infty} \frac{\mu\left(B_{k}\right)}{\mu\left(A_{k}\right)}=\lambda \quad \text { and } \quad \lim _{k \rightarrow \infty} \frac{\mu\left(B_{k}^{\prime}\right)}{\mu\left(A_{k}\right)}=(1-\lambda)
$$

taking into account the fact that $x$ and $y$ are Lebesgue points for $u$, we thus obtain from the identity (5)

$$
\lim _{k \rightarrow \infty} u_{A_{k}}=\lambda u(x)+(1-\lambda) u(y) .
$$

Observe that

$$
\begin{aligned}
& \lim _{k \rightarrow \infty} f_{B_{k}}\left|u(z)-u_{A_{k}}\right| \mathrm{d} \mu_{z}=\left|u(x)-\lim _{k \rightarrow \infty} u_{A_{k}}\right|=(1-\lambda)|u(x)-u(y)|, \\
& \lim _{k \rightarrow \infty} f_{B_{k}^{\prime}}\left|u(z)-u_{A_{k}}\right| \mathrm{d} \mu_{z}=\left|u(y)-\lim _{k \rightarrow \infty} u_{A_{k}}\right|=\lambda|u(x)-u(y)| .
\end{aligned}
$$

Hence from

$$
f_{A_{k}}\left|u(z)-u_{A_{k}}\right| \mathrm{d} \mu_{z}=\frac{\mu\left(B_{k}\right)}{\mu\left(A_{k}\right)} \int_{B_{k}}\left|u(z)-u_{A_{k}}\right| \mathrm{d} \mu_{z}+\frac{\mu\left(B_{k}^{\prime}\right)}{\mu\left(A_{k}\right)} \int_{B_{k}}\left|u(z)-u_{A_{k}}\right| \mathrm{d} \mu_{z},
$$

we conclude that

$$
\lim _{k \rightarrow \infty} f_{A_{k}}\left|u(z)-u_{A_{k}}\right| \mathrm{d} \mu_{z}=2 \lambda(1-\lambda)|u(x)-u(y)|
$$


Using now the fact that $x$ and $y$ are also Lebesgue points for $g^{p}$, we have

$$
\begin{aligned}
\lim _{k \rightarrow \infty} \int_{A_{k}} g(z)^{p} \mathrm{~d} \mu_{z} & \leqslant \lim _{k \rightarrow \infty} \frac{\mu\left(B_{k}\right)}{\mu\left(A_{k}\right)} \int_{B_{k}} g(z)^{p} \mathrm{~d} \mu_{z}+\lim _{k \rightarrow \infty} \frac{\mu\left(B_{k}^{\prime}\right)}{\mu\left(A_{k}\right)} \int_{B_{k}^{\prime}} g(z)^{p} \mathrm{~d} \mu_{z} \\
& =\left(\lambda g(x)^{p}+(1-\lambda) g(y)^{p}\right) \\
& \leqslant(g(x)+g(y))^{p} .
\end{aligned}
$$

Since we clearly have $\lim _{k \rightarrow \infty} \operatorname{diam}\left(A_{k}\right)=d(x, y)$, we obtain from (4), (6) and (7)

$$
\begin{aligned}
2 \lambda(1-\lambda)|u(x)-u(y)| & =\lim _{k \rightarrow \infty} f_{A_{k}}\left|u(z)-u_{A_{k}}\right| \mathrm{d} \mu_{z} \\
& \leqslant \lim _{k \rightarrow \infty}\left(C^{\prime} \operatorname{diam} A_{k}\right)\left(f_{A_{k}} g^{p} \mathrm{~d} \mu\right)^{1 / p} \\
& \leqslant C^{\prime} d(x, y)(g(x)+g(y)) .
\end{aligned}
$$

This implies the inequality

$$
|u(x)-u(y)| \leqslant \frac{C^{\prime}}{2 \lambda(1-\lambda)} d(x, y)(g(x)+g(y)) \leqslant\left(2+K+K^{-1}\right) d(x, y)(g(x)+g(y)) .
$$

Now observe that

$$
\frac{1}{\lambda(1-\lambda)}=\frac{1}{\ell}(1+\ell)^{2}=\left(\ell+2+\frac{1}{\ell}\right) \leqslant\left(2+K+K^{-1}\right)
$$

hence if one sets $C:=\frac{1}{2}\left(2+K+K^{-1}\right) C^{\prime}$, then we have

$$
|u(x)-u(y)| \leqslant C d(x, y)(g(x)+g(y)) .
$$

Observe that in the proof of the theorem, we only use the inequality (4) for sets $A$ which are disjoint unions of two small balls.

Final remark. - The main theorem would be false with (2) replacing (4). Observe that the main difference between the inequalities (2) and (4) is that in (4), one considers averages over arbitrary sets whereas (2) is restricted to balls.

However, using the maximal function, one may obtain a Hajłasz type inequality (1) from a Poincaré inequality on balls (2). More precisely the following result is known:

THEOREM 5. - Let $X$ be a doubling space and $p>0$. If $(u, g)$ satisfies a $(1, p)$-Poincaré inequality (2), then we have a.e.

$$
|u(x)-u(y)| \leqslant C d(x, y)\left(M_{2 \sigma d(x, y)}\left(g^{p}(x)\right)^{1 / p}-M_{2 \sigma d(x, y)}\left(g^{p}(y)\right)^{1 / p}\right),
$$

where

$$
M_{R}(h(x))=\sup _{0<r<R} f_{\mathrm{B}(x, r)}|h| \mathrm{d} \mu .
$$

This result is Theorem 3.2 in [5], a particular case was also obtained in [2], Example 6.

\footnotetext{
${ }^{1}$ It is in fact more accurate to say that the pair $(u, g)$ is an analog of the pair $(u, M(|\nabla u|))$ where $M()$ is the maximal operator.
} 


\section{Troyanov, V. Gol'dshtein}

\section{References}

[1] Franchi B., Hajłasz P., Koskela P., Definitions of Sobolev classes on metric spaces, Ann. Inst. Fourier 49 (1999) 1903-1924.

[2] Franchi B., Lu G., Wheeden R., A relationship between Poincaré-type inequalities and representation formulas in spaces of homogeneous type, Int. Math. Res. Notices 1 (1996) 1-14.

[3] Gol'dshtein V.M., Troyanov M., Axiomatic theory of Sobolev spaces, Expositiones Mathematicae (to appear).

[4] Hajłasz P., Sobolev spaces on an arbitrary metric space, Potent. Anal. 5 (4) (1996) 403-415.

[5] Hajłasz P., Koskela P., Sobolev met Poincaré, Mem. Amer. Math. Soc. 145 (688) (2000).

[6] Kinnunen J., Martio O., The Sobolev capacity on metric spaces, Ann. Acad. Sci. Fennicae, Series Mathematica 21 (1996) 367-382.

[7] Koskela P., MacManus P., Quasiconformal mappings and Sobolev spaces, Studia Math. 131 (1) (1998) 1-17. 